\title{
Anti-herpes Simplex Virus Target of an Acidic Polysaccharide, Nostoflan, from the Edible Blue-Green Alga Nostoc flagelliforme
}

\author{
Kenji Kanekiyo, ${ }^{a}$ Kyoko Hayashi, ${ }^{a}$ Hiroyuki Takenaka, ${ }^{b}$ Jung-Bum Lee, ${ }^{a}$ and Toshimitsu Hayashi ${ }^{*}, a$ \\ ${ }^{a}$ Graduate School of Medicine and Pharmaceutical Sciences, University of Toyama; 2630 Sugitani, Toyama 930-0194, \\ Japan: and ${ }^{b}$ Mac Gifu Research Institute; 4-15 Akebono, Gifu 500-8148, Japan. \\ Received April 12, 2007; accepted May 7, 2007
}

The acidic polysaccharide nostoflan was previously isolated as an antiviral component from the terrestrial alga Nostoc flagelliforme. In the present study, we examined the target for its anti-herpes simplex virus type 1 action. In time-of-addition experiments, the most sensitive stage of viral replication to nostoflan was found to be early events, including the virus binding and/or penetration processes. In order to determine what extent nostoflan may be involved in these processes, virus binding and penetration assays were separately performed. The results indicated that the inhibition of virus binding to but not penetration into host cells was responsible for the antiherpetic effect induced by nostoflan. Our study suggests that nostoflan may be a potential antiherpes agent.

Key words Nostoc flagelliforme; herpes simplex virus; antiviral target; viral binding process

Several synthesized nucleoside analogs are available for the treatment or suppression of herpes simplex virus (HSV) infections. Acyclovir (ACV) is a representative of these analogs. ${ }^{1)}$ Recently, a number of reports have demonstrated a decline of immune function due to age, stress or medical interventions. When the immune defense mechanism of a host is impaired, serious infections by non- or low-pathogenic microorganisms frequently occur, and drug-resistant pathogens might easily be generated. In fact, ACV-resistant HSV has been isolated from immunocompromised persons. ${ }^{2-5)}$ Thus, the development of novel inhibitors of HSV replication with different mechanisms of action is still required.

We have recently reported on the isolation of a novel antiviral component, named nostoflan, from the blue-green alga Nostoc flagelliforme. Nostoflan showed a potent inhibitory effect on HSV type 1 (HSV-1). ${ }^{6}$ In the present study, we investigated the antiviral target of this polysaccharide in order to determine its potential as an antiherpetic agent.

\section{MATERIALS AND METHODS}

Materials Nostoflan was prepared from N. flagelliforme collected in China as described previously. $\left.{ }^{6}\right)$ Dextran sulfate (MW 500000) was purchased from Sigma-Aldrich Corp. (St. Lous, Mo, U.S.A.).

Cells and Virus Vero cells were grown in Eagle's minimal essential medium (MEM) (Nissui Pharmaceutical Co., Ltd., Tokyo, Japan) supplemented with $5 \%$ fetal bovine serum (FBS). HSV-1 (HF strain) was propagated and plaqueassayed on Vero cell monolayers.

Antiviral Activity For the determination of anti-HSV-1 activity, plaque assay was employed as described previously. ${ }^{7)}$ Briefly, Vero cell monolayers were infected with HSV-1 at room temperature for $1 \mathrm{~h}$. The monolayers were washed three times with phosphate-buffered saline (PBS) and incubated in maintenance medium (MEM plus 2\% FBS) at $37^{\circ} \mathrm{C}$. The different concentrations of nostoflan were added to the monolayers at the indicated time. Virus yields were determined by plaque assay on Vero cell monolayers after 1-d incubation. Antiviral activity was expressed as the $50 \%$ inhibitory concentration $\left(\mathrm{IC}_{50}\right)$, which was the lowest compound concentration that reduced plaque number by $50 \%$ in the treated cultures compared with untreated control ones.

Time-of-Addition Experiments Vero cell monolayers were infected with HSV-1 at 10 plaque-forming units (PFU) per cell. Nostoflan $(0,0.1,1,10,100 \mu \mathrm{g} / \mathrm{ml})$ was added for $3 \mathrm{~h}$ before viral infection, during viral infection for $1 \mathrm{~h}$, during viral infection and throughout the incubation for $24 \mathrm{~h}$, immediately after infection for $24 \mathrm{~h}$, from 2 to $24 \mathrm{~h}$ postinfection (p.i.), from 4 to $24 \mathrm{~h}$ p.i., or from 6 to $24 \mathrm{~h}$ p.i. At $24 \mathrm{~h}$ p.i., the cell cultures were harvested and subjected to plaque assay.

Assay for Virus Adsorption The effect of nostoflan on HSV-1 adsorption to host cells was evaluated by an infectious center assay. ${ }^{8)}$ Briefly, Vero cell suspension $\left(4 \times 10^{6}\right.$ cell $/ \mathrm{ml}), \mathrm{HSV}-1$ at $1 \mathrm{PFU}$ per cell, and different concentrations of nostoflan or dextran sulfate were precooled at $4{ }^{\circ} \mathrm{C}$ for $3 \mathrm{~h}$ before mixing. The mixtures were incubated at $4{ }^{\circ} \mathrm{C}$ for $1 \mathrm{~h}$ to allow the virus binding to cells and to prevent the virus from penetrating into cells. After the adsorption period, unbound virus and free compound were removed by washing three times with ice-cold PBS. The cell pellets were diluted serially with ice-cold PBS and added to Vero cell monolayers, which were overlaid with maintenance medium containing $0.5 \%$ methylcellulose (MC) to be plaque-assayed.

Assay for Virus Penetration Virus penetration into host cells was evaluated according to the method reported by Huang and Wagner ${ }^{9)}$ and modified by Highlander et al. ${ }^{10)}$ Vero cell monolayers precooled at $4^{\circ} \mathrm{C}$ for $3 \mathrm{~h}$ were infected with HSV-1 (approximately $100 \mathrm{PFU} /$ well) at $4{ }^{\circ} \mathrm{C}$ for $1 \mathrm{~h}$ in the absence of nostoflan or dextran sulfate. After washing three times with ice-cold PBS, maintenance medium containing different concentrations of nostoflan or dextran sulfate was added to the cell monolayers, the temperature of which was shifted to $37^{\circ} \mathrm{C}$. At $0,0.5,1,2,3$ and $6 \mathrm{~h}$ of incubation at $37^{\circ} \mathrm{C}$, unpenetrated viruses were inactivated by treating the cell monolayers with $40 \mathrm{~mm}$ citrate buffer $(\mathrm{pH} 3.0$ ) for $1 \mathrm{~min}$. The cell monolayers were overlaid with maintenance medium containing $0.5 \% \mathrm{MC}$ to be plaque-assayed.

Assay for Virus Inactivation The virucidal activity of nostoflan was determined as follows: HSV-1 suspension con- 
taining $4 \times 10^{5} \mathrm{PFU}$ per $\mathrm{ml}$ was mixed with an equal volume of medium containing $0,2,20,200$ and $2000 \mu \mathrm{g}$ of nostoflan per $\mathrm{ml}$. The mixtures were incubated for $1.5 \mathrm{~h}$ at $37^{\circ} \mathrm{C}$, diluted 100-fold with PBS to eliminate the effect of nostoflan on virus replication, and subjected to plaque formation to determine the remaining infectivity.

Analysis of Radiolabeled HSV-1-Specific Glycoprotein Vero cell monolayers in $35-\mathrm{mm}$ dishes were infected with HSV-1 at 10 PFU per cell at room temperature for $1 \mathrm{~h}$, washed three times with PBS, and replenished with methionine-free MEM (Flow Laboratories, Irvine, U.K.). The infected cells were radiolabeled during $2-8 \mathrm{~h}$ postinfection with $10 \mu \mathrm{Ci}$ of $\operatorname{Tran}{ }^{35} \mathrm{~S}-$ label $(43.47 \mathrm{TBq} / \mathrm{mm}$; ICN Biomedicals, Costa Mesa, CA, U.S.A.). Different concentrations of nostoflan were added to the cells at the same time as viral infection and throughout the incubation thereafter or immediately after viral infection. The infected cells were harvested at $8 \mathrm{~h}$ postinfection and extracted with cell lysis buffer $(0.05 \mathrm{~m}$ Tris- $\mathrm{HCl}, \mathrm{pH} 7.0,0.15 \mathrm{M} \mathrm{NaCl}, 1 \%$ SDS, $1 \%$ Triton $\mathrm{X}-100)$. The cell lysates ( $50 \mu \mathrm{g}$ of protein) were treated with rabbit antibody prepared against synthesized oligopeptide of HSV-1 glycoprotein D (gD) as reported previously ${ }^{7)}$ and Protein A Sepharose 4B Fast Flow (Pharmacia Biotech AB, Uppsala, Sweden) overnight at $4{ }^{\circ} \mathrm{C}$ with rotating. The immunoprecipitates were subjected to SDS-PAGE $(8 \%$ acrylamide gel). After electrophoresis, the gels were soaked in $1 \mathrm{M}$ sodium salicylate for $30 \mathrm{~min}$, dried, and exposed to X-ray films. ${ }^{11)}$

Statistical Analysis The data are expressed the mean \pm S.D. from triplicate assays, and were analyzed by Student's $t$ test.

\section{RESULTS}

Effect of Time of Addition of Nostoflan on HSV-1 Replication In order to determine the most sensitive phase of HSV-1 replication to nostoflan, time-of-addition experiments were performed. In these experiments, Vero cells were infected with HSV-1 at a high multiplicity of infection of 10, where all the cells were infected with the virus as determined by infectious center assay (data not shown). As shown in Fig. 1 , nostoflan suppressed virus production most efficiently in a dose-dependent manner when added at the same time of virus infection and throughout the incubation thereafter. The presence of nostoflan only during viral infection for $1 \mathrm{~h}$ also produced relatively potent inhibitory effects on HSV-1 replication. However, the compound was much less effective and showed no dose dependence when the host cells were pretreated for $3 \mathrm{~h}$ or it was added at various times after viral infection.

Effect of Nostoflan on Virus Adsorption to Host Cells From the time-of-addition experiments described above, the most sensitive phase of HSV-1 replication to nostoflan was suggested to be early events including virus binding to the receptor on host cell membranes and/or virus penetration into host cells by fusion between the virus envelope and cell membrane. At first, the effect of nostoflan on HSV-1 adsorption to host cells was evaluated by infectious center assay, which determined the number of cells binding the virus particles at low temperature $\left(4^{\circ} \mathrm{C}\right)$ in the presence of different concentrations of the compound. As shown in Fig. 2, nos-
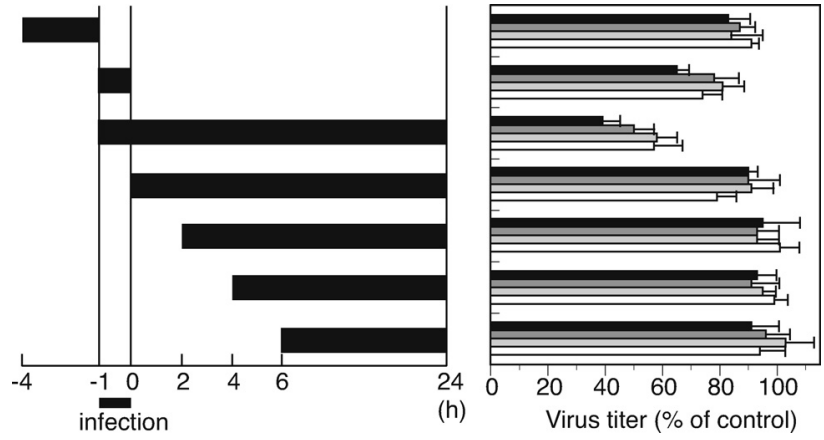

Fig. 1. Effects of Time of Addition of Nostoflan on HSV-1 Replication

Vero cells were infected with HSV-1 at 10 PFU/cell. Nostoflan was added to the culture medium at the times shown in the left panel. Briefly, sample was added before viral infection for $3 \mathrm{~h}$, during infection for $1 \mathrm{~h}$, during infection and throughout the incubation thereafter, immediately after infection, at $2 \mathrm{~h}$ postinfection (p.i.), at $4 \mathrm{~h}$ p.i. or at $6 \mathrm{~h}$ p.i. at the concentrations of 0.1 (white bar), 1 (light gray bar), 10 (gray bar), and 100 (black bar) $\mu \mathrm{g} / \mathrm{ml}$. The cell cultures were harvested at $24 \mathrm{~h}$ p.i. for titration of virus yield. Data are presented as the mean \pm S.D. from triplicate assays.

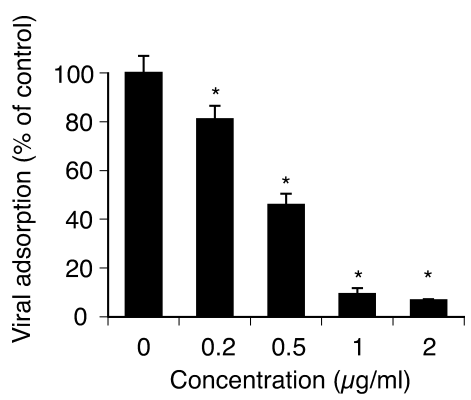

Fig. 2. Effect of Nostoflan on HSV-1 Adsorption to Host Cells

Vero cell suspensions were infected with HSV-1 (1 PFU/cell) at $4{ }^{\circ} \mathrm{C}$ in the presence of $0,0.2,0.5,1$, and $2 \mu \mathrm{g} / \mathrm{ml}$ nostoflan. The cells binding virus particles were detected by infectious center assay as described in the Materials and Methods. The asterisk (*) indicates a significant difference between control and nostoflan-treated cells, $* p<0.01$.

toflan interfered significantly with virus adsorption in a dosedependent manner. Dextran sulfate also showed approximately 15 and $85 \%$ inhibition of virus adsorption at 0.2 and $1 \mu \mathrm{g} / \mathrm{ml}$, respectively, as compared with control without sample.

Effect of Nostoflan on Virus Penetration into Host Cells The virus penetration process is one possible target of nostoflan, as suggested by the time-of-addition experiments. After HSV-1 was adsorbed to Vero cells under the conditions that caused no virus penetration, the kinetics of virus penetration in the presence of different concentrations of nostoflan were studied for $6 \mathrm{~h}$ postinfection. Nostoflan showed no significant dose-dependent inhibition of virus internalization even at the high concentration of $1000 \mu \mathrm{g} / \mathrm{ml}$ (data not shown). However, dextran sulfate showed approximately 10 , 50 and $75 \%$ inhibition of virus penetration at $0.2,1$ and $10 \mu \mathrm{g} / \mathrm{ml}$, respectively, as compared with control.

Effect of Nostoflan on HSV-1 Inactivation When HSV1 was preincubated for $1.5 \mathrm{~h}$ in the presence of $1,10,100$, and $1000 \mu \mathrm{g} / \mathrm{ml}$ nostoflan, the infectivity of the virus was only reduced to approximately $85 \%$ of control even at the highest concentration of $1000 \mu \mathrm{g} / \mathrm{ml}$ (data not shown). Thus, nostoflan might have no marked effect on direct virus inactivation.

Effect of Nostoflan on HSV-1-Specific Protein Synthesis As mentioned above, nostoflan prevented HSV-1 from binding to host cells. In order to confirm that this antiviral action 


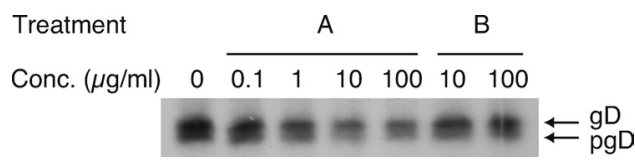

Fig. 3. Effect of Nostoflan on Viral Protein Synthesis

HSV-1-infected cells radiolabeled with Tran ${ }^{35} \mathrm{~S}$-label were harvested at $8 \mathrm{~h}$ p.i. The cell lysates were subjected to immunoprecipitation with anti-HSV-1 gD antibody followed by SDS-PAGE. The cells were treated with $0.1,1,10$, and $100 \mu \mathrm{g} / \mathrm{ml}$ nostoflan during virus infection and throughout the incubation thereafter (A), or, with 10 and $100 \mu \mathrm{g} / \mathrm{ml}$ nostoflan immediately after infection (B).

of the compound might be reflected in the intracellular events thereafter, HSV-1 gD protein, an essential glycoprotein, was detected in the cell cultures treated with nostoflan. The cell cultures were treated during radiolabeling with the compound by adding from the point of virus infection (Fig. 3A), or, immediately after infection (Fig. 3B). The antibody used detected both the precursor (pgD) and mature $(\mathrm{gD})$ forms of $\mathrm{gD}$. In the cells treated with nostoflan from the time of virus infection, the amounts of $\mathrm{gD}$ and $\mathrm{pgD}$ were dose-dependently decreased in the range of 0.1 to $10 \mu \mathrm{g} / \mathrm{ml}$ nostoflan. However, when nostoflan treatment was started after virus infection, the synthesis of the glycoprotein was less inhibited as compared with that in the cells treated with the compound during virus infection.

\section{DISCUSSION}

The acidic polysaccharide nostoflan isolated from the terrestrial alga $N$. flagelliforme prevented virus replication in a similar way to sulfated polysaccharides, another class of acidic polysaccharides. Many kinds of synthesized and algae-derived sulfated polysaccharides such as dextran sulfate, fucoidan, spirulan, and hornan have been reported to be antiviral components. ${ }^{12-19)}$ Some sulfated polysaccharides are known to inhibit both virus binding and penetration processes in the early phase of virus-cell interactions. In the present study, dextran sulfate was shown to have inhibitory effects on these two processes. We have also recently reported that a sulfated polysaccharide from a diatom showed dose-dependent inhibition of HSV-1 binding to and penetration into host cells. ${ }^{20)}$ On the other hand, nostoflan, which has no sulfate group, was found to exert an inhibitory effect on the virus binding process but not on the virus penetration process that occurs immediately after virus binding. Whether these differences in antiviral targets between nostoflan and sulfated polysaccharides depend solely on the presence of sulfate residues remains unclear. The cells synthesized less amounts of HSV-specific $\mathrm{gD}$ in the presence of nostoflan during virus infection as compared with those treated with the compound after virus infection, when the virus binding process should have been finished. HSV-1 gD is an essential glycoprotein which is required for the fusion between virus and host cell. ${ }^{21)}$ Therefore, the level of $\mathrm{gD}$ in HSV-infected cells can be considered to reflect the level of viral replication. Since the presence of nostoflan after virus binding exerted almost no effect on the synthesis of HSV-1 gD, the main target of the compound was suggested not to be the intracellular step of viral replication. From these results, it was found that the anti-HSV target of nostoflan is quite different from that of ACV, which inhibits viral DNA synthesis in infected cells. $^{22)}$

In general, polysaccharides including sulfated polysaccharides are known as immunomodulating agents. ${ }^{23-26)}$ We have recently confirmed the effect of nostoflan on the stimulation of immune response in influenza virus-infected mice (manuscript in preparation). These characteristics might be favorable to the suppression of HSV replication in the body, especially in immunocompromised hosts.

In summary, nostoflan was found to have a unique antiherpetic target in the in vitro HSV-1 replication system. Further investigation of the polysaccharide would be useful to overcome the problems that might limit the application of antiherpetic nucleoside analogs, including ACV.

\section{REFERENCES}

1) Crumpacker C. S., Schnipper L. E., Zaia J. A., Levin M. J., Antimicrob. Agents Chemother, 15, 642-645 (1979).

2) Burns W. H., Santos G. W., Saral R., Laskin O. L., Lietman P. S. McLaren C., Barry D. W., Lancet, 319, 421-423 (1982).

3) Erlich S. K., Mills J., Chatis P., Mertz G. J., Busch D. F., Follansbee S. E., Grant R. M., Crumpacker C. S., New Engl. J. Med., 320, 293-296 (1988).

4) Chatis P. A., Crumpacker C. S., Virology, 180, 793-797 (1991).

5) Posavad C. M., Koelle D. M., Shaughnessy M. F., Corey L., Proc. Natl. Acad. Sci. U.S.A., 94, 10289-10294 (1997).

6) Kanekiyo K., Lee J.-B., Hayashi K., Takenaka H., Hayakawa Y., Endo S., Hayashi T., J. Nat. Prod., 68, 1037-1041 (2005).

7) Hayashi K., Hayashi T., Ujita K., Takaishi Y., J. Antimicrob. Chemother, 37, 759-768 (1996).

8) Andries K., Dewindt B., Snoecks J., Willebrords R., Van Eemeren K., Stokbroekx R., Janssen P. A. J., Antimicrob. Agents Chemother., 36, 100-107 (1992).

9) Huang A. S., Wagner R. R., Proc. Soc. Exp. Biol. Med., 116, 863869 (1964).

10) Highlander S. L., Sutherland S. L., Gage P. J., Johnson D. C., Levine M., Glorioso J. C., J. Virol., 61, 3356-3364 (1987).

11) Sambrook J., Fritsch E. F., Maniatis T., "Molecular Cloning: a Laboratory Manual," Cold Spring Harbor Laboratory, Cold Spring Harbor, N.Y., 1989.

12) Baba M., Pauwels R., Balzarini J., Arnout J., Desmyter J., De Clercq E., Proc. Natl. Acad. Sci. U.S.A., 85, 6132-6136 (1988).

13) Mitsuya H., Looney D. J., Kuno S., Ueno R., Wong-Staal F., Broder S., Science, 240, 646-649(1988).

14) Huheihel M., Ishanu V., Tal J., Arad S. M., J. Biochem. Biophys. Methods, 50, 189-200 (2002).

15) Lee J.-B., Hayashi K., Hashimoto M., Nakano T., Hayashi T., Chem. Pharm. Bull., 52, 1091-1094 (2004).

16) Hayashi K., Hayashi T., Kojima I., AIDS Res. Hum. Retroviruses, 12 1463-1471 (1996).

17) Lee J.-B., Hayashi T., Hayashi K., Sankawa U., J. Nat. Prod., 63, $136-138$ (2000)

18) Hoshino T., Hayashi T., Hayashi K., Hamada J., Lee J.-B., Sankawa U., Biol. Pharm. Bull., 21, $730-734$ (1998).

19) Preeprame S., Hayashi K., Lee J.-B., Sankawa U., Hayashi T., Chem. Pharm. Bull., 49, 484-485 (2001).

20) Lee J.-B., Hayashi K., Hirata M., Kuroda E., Suzuki E., Kubo Y., Hayashi T., Biol. Pharm. Bull., 29, $2135-2139$ (2006).

21) Ligas M. W., Johnson D. C., J. Virol., 62, 1486-1494 (1988).

22) Miller W. H., Miller R. L., J. Biol. Chem., 255, 7204-7207 (1980).

23) Itoh H., Noda H., Amano H., Ito H., Anticancer Res., 15, 1937-1948 (1995).

24) Okai Y., Higashi-Okai K., Ishizaka S., Ohtani K., Matsui-Yuasa I., Yamashita U., J. Sci. Food Agric., 76, 56-62 (1998).

25) Shan B. E., Yoshida Y., Kuroda E., Yamashita U., Int. J. Immunopharmacol., 21, 59-70 (1999).

26) Liu J. N., Yoshida Y., Wang M. Q., Okai Y., Yamashita U., Int. J. Immunopharmacol., 19, 135-142 (1997). 